\title{
GRAVITATIONAL COLLAPSE AND THE \\ FORMATION OF THE SOLAR NEBULA
}

\author{
R. B. LARSON
}

Dept. of Astronomy, Yale University, New Haven, Conn., U.S.A.

\begin{abstract}
Most theories of the origin of the solar system begin by assuming that, at the time of its formation or shortly afterwards, the Sun somehow acquired a disc-like 'solar nebula' in which the planets later formed by accretion. The presently available gravitational collapse calculations, while not yet sufficiently sophisticated to delineate in detail the processes involved, at least support the plausibility of this idea and suggest that the solar nebula may have formed by the accretion or capture of leftover protostellar material into orbit around the early Sun. Infall of matter into the disc probably continued for roughly $10^{5}$ to $10^{6} \mathrm{yr}$, and may have produced transient strong heating effects through the thermalization of the infall energy in shock fronts. It seems likely that the satellite systems formed in a basically similar way through the capture of residual solid matter into orbit around the planets.

A more complete discussion of gravitational collapse and the formation of the solar system has been given in The Origin of the Solar System, ed. H. Reeves, p. 142. (Paris: CNRS).
\end{abstract}

